\title{
Hipóteses de Trabalho: Disciplinando o Raciocínio*
}

Tom O. Miller**

\author{
MILLER, T.O. Hipóteses de trabalho: disciplinando o raciocínio. Revista do Museu de \\ Arqueologia e Etnologia, São Paulo, 19: 3-12, 2009.
}

Resumo: Após considerações teóricas em torno do raciocínio científico na elaboração e teste de hipóteses, o autor, manipulando os elementos da Lógica Simbólica para gerar hipóteses alternativas, considera as vantagens do uso desta como um instrumento de exame e esclarecimento das relações entre os fenômenos em estudo. As suas conclusões, embora inseridas no contexto da Arqueologia, valem para qualquer ciência social.

Palavras chaves: Lógica do método científico - Geração de hipóteses múltiplas - Arqueologia - Lógica Simbólica.

\begin{abstract}
Arqueologia Moderna procura aumentar a gama de informações disponiveis sobre atividades humanas num passado não registrado, que sejam confirmáveis dentro do registro arqueológico, e explanar tais atividades como casos específicos de regularidades ou causas mais gerais (Miller 1978; 1979). Para isto, busca-se aprimorar a metodologia, agrupando declarações descritivas para encontrar padrões de regularidades subjacentes
\end{abstract}

${ }^{*}$ E) Este trabalho baseia-se numa versão anterior apresentada na XIIa Reunião da Associação Brasileira de Antropologia, Rio de Janeiro, julho de 1980. Originalmente o trabalho era para ser publicado no primeiro número de uma projetada Revista do Museu "Câmara Cascudo", revista esta que não saiu. $\mathrm{O}$ manuscrito ficou na prateleira até ser redescoberto, recentemente. Apesar desta defasagem, acreditamos que o trabalho merece ser publicado porque o assunto continua atual.

$\left.{ }^{* *}\right)$ Professor aposentado do Departamento de Ciências Sociais (agora Departamento de Antropologia) e do Museu "Câmara Cascudo" da Universidade Federal do Rio Grande do Norte.
(Miller 1978a; ver também Barbosa 1976; Brochado 1977 e Lima 1987, para exemplos da "Nova Arqueologia" no Brasil).

A padronização começa pela parte descritiva, através das declarações descritivas que realçam as semelhanças e diferenças nos fenômenos descritos. No nível analítico, aplicase a teoria aos fenômenos (objetos, relações e atividades descritos) para mostrar como estes fenômenos exibem propriedades analiticamente determináveis e, portanto, utilizáveis para explicar certos aspectos do sistema do qual fazem ou fizeram parte.

Verificamos ou refutamos as nossas proposições ao construir modelos que podem ser testados contra os dados.

Uma teoria, sendo baseada numa série de construções analíticas, não é testada, para ver se correta, contra dados empíricos. Em vez disto, se usa um modelo concreto da teoria para testar a aplicabilidade da mesma ao mundo real (...) Uma falha do modelo ao exibir propriedades inerentes ao mundo real 
(...) implica ou que a teoria precisa ser reformulada, ou que uma identificação inválida dos primitivos termos da teoria com o mundo real tenha sido feita, mas não que a teria deixa de ser correta. Embora a geometria de duas dimensões, estritamente falando, não seja apropriada ao mundo tridimensional, não há nada incorreto na geometria duo-dimensional como teoria (Read e Le Blanc 1978: 310).

Utilizando a teoria de sistemas como uma teoria abstrata, podemos construir modelos de partes do sistema, ou do sistema como um todo, para daí derivar implicações testáveis contra os dados do mundo real. Uma vez que os modelos sejam concretizados, podemos tornar explícitas as pressuposições que antes eram implícitas e, portanto, não examinadas, revelando falhas potenciais no raciocínio e na coleta de dados. Isto permite também que implicações adicionais possam ser testadas.

Sendo assim, convém elaborar um conjunto preliminar de hipóteses de trabalho, transformando-as em modelos concretos, antes de fazer o trabalho de campo, pois as implicações deriváveis podem forçar e, provavelmente, forçarão um reexame ou reformulação dos métodos e dos locais de coleta de dados.

Em 1969 Hole e Heizer observaram:

Ao examinar subsistemas culturais dirigimos a nossa atenção pela primeira vez aos seres humanos, não como individuos, mas como membros de comunidades outrora vivas. No entanto, não podemos compreender (a vida de) essa gente em todas as suas facetas. Podemos dizer pouco sobre a sua politica ou teologia, mas podemos perceber alguns dos meios para reconstituir a maneira pela qual se organizaram. Todas as coisas são constituidas de partes organizadas; é uma das nossas tarefas como cientistas sociais tentar descobrir os principios de organização que de amontoados de gente fazem sociedades viáveis. Como arqueólogos, teremos que tentar reconstituir a organização do povo cujos resquicios podemos manusear, contar, medir e desenhar. Torna-se cada vez mais patente que os princípios de organização são as chaves básicas para a nossa compreensão de qualquer classe de fenômenos, inclusive a de gente. A esta posição assumem os teóricos de sistemas gerais, e à qual, há muitos anos, fazem eco, de maneiras variadas, os antropólogos.

$\dot{E}$ provavelmente afortunado que a organização emergiu como o ponto de enfoque de estudos de comportamento, porque os arqueólogos conseguem encontrar na pré-história informações que dizem respeito à maneira pela qual sítios e artefatos ocorrem, fornecem indicios da organização da sociedade que os deixou (Hole e Heizer 1969: 269-70).

Vejamos bem: nas ponderações de Hole e Heizer, o enfoque não está nas coisas em si e sim nas relações entre as coisas. Isto tem que resultar em hipóteses de trabalho na forma de modelos que expressam tais relações de maneira simples e que deixam claras as pressuposições subjacentes para exame.

Apesar do florescimento de formulações teóricas na Arqueologia após a década de 1960, ainda se percebe uma contradição no sentido de que alguns arqueólogos fazem construções teóricas que não são testadas, porque, ou não têm tempo, ou não têm verba para tal. Outros, por sua vez, fazem o seu trabalho de maneira meramente indutiva, quando não, simplesmente ritualizada, repetindo as observações descritivas do conteúdo de sítios e componentes e comparando-as com resultados de outras escavações similarmente estruturadas em outros sítios, considerados "típicos" não porque representam fielmente a média da cultura examinada, mas porque simplesmente foram escavados anteriormente. $\mathrm{O}$ que se observa de fato é que há uma falta de entrosamento entre teoria e método na divulgação de pesquisas arqueológicas.

Para começar, com relação ao item “verbas", este não é contemplado quando se trata de grandes equipes testarem teorias relacionadas com o funcionamento de sistemas culturais inteiros. Quando, porém, a finalidade é atender à legislação que 
protege o patrimônio pré-histórico, ou seja, os projetos de arqueologia de salvamento, as verbas apresentam-se num contínuo aumento. Aliás, é neste campo que ocorre atualmente, paralelamente à saturação do mercado acadêmico, uma carência de pessoal qualificado.

Adicionalmente, tem-se considerado que testar hipóteses em projetos pequenos ou lineares, como os de salvamento seria um tanto difícil. Ao se examinar melhor o tema, porém, percebe-se que esse não é necessariamente o caso. Tudo vai depender do alcance e da manipulação das hipóteses de trabalho.

Primeiro, precisa-se distinguir dois tipos de projetos de salvamento (Gumerman 1973). Precisamente, temos os projetos que envolvem "grandes massas de território, e nos quais o arqueólogo tem ao menos vários meses de sobreaviso antes que a modificação da paisagem comece" (ibidem, p. 291), tempo suficiente para desenvolver os trabalhos. Esses projetos são relacionados mais frequentemente ao represamento de águas fluviais, sistemas de irrigação ou operações de mineração na superfície. Nada impede que se elaborem hipóteses a serem testadas nesse tipo de operação.

O outro tipo é o pequeno projeto, frequentemente linear, que envolve menos tempo e área mais restrita. Nesse tipo de projeto a integração método/teoria torna-se mais difícil a não ser no caso de projetos de direito de passagem para estradas, linhas de transmissão e de encanamento, que podem abranger uma distância considerável, atravessando diversos ambientes. Aqui se deve salientar a necessidade de se prestar atenção à diversidade de atividades encontradas nas áreas de borda ou transição, entre zonas ecológicas maiores, pois as populações humanas tendem a concentrar uma maior variedade de técnicas adaptativas nessas zonas de "margem" ou tensão ecológica, ao se aproveitarem da maior variedade de populações de plantas e animais e outros recursos.

Como frisou Redman, a abordagem sistêmica tem muito a contribuir para ajudar a entender as relações entre os fenômenos:
Há várias contribuições importantes da abordagem sistêmica às investigações arqueológicas. A mais ampla é a ênfase na grande complexidade dos processos e da organização culturais. Sistemas são complexos (...) Os aspectos primários desta complexidade são o inter-relacionamento dos elementos no sistema e a relação estreita do sistema com o seu ambiente (...) Coleção e análise de dados têm que ser feitas de uma maneira que realce a variabilidade no registro arqueológico e providencie uma amostra da gama completa desta variação. Sítios individuais não são uma amostra adequada dentro de uma armação sistêmica, e resumos de sitios individuais não podem ser feitos a partir de um exposição limitada do sítio. Investigações extensivas que estudam uma amostra representativa da população inteira são necessárias (...)

A natureza holista (total) da abordagem sistêmica permite ao pesquisador prosseguir numa investigação controlada do seu assunto sem que se exija que possa manter certas variáveis constantes (uma façanha difícil ao estudar culturas). Ao estudar o que se pode reconhecer da estrutura e das respostas do sistema a vários inputs, é possível inferir a natureza do sistema. O expressar desta estrutura na forma de um diagrama força o pesquisador a se tornar explicito e preciso sobre a maneira pela qual os vários elementos do sistema reagiriam a vários estímulos (Redman 1973: 18-19).

E também, como Wittfogel (1957) e Steward (1955b) demonstraram, existem sequências que tendem a se repetir uma vez iniciadas - e ainda não temos mapeado tais "trilhas", "entroncamentos" e quaisquer "ramais" nos processos socioculturais, nem as suas condições-limites com as coerções associadas. Em 1955 os arqueólogos americanos tentaram uma classificação inicial (Wauchope 1955), o que também foi feito em 1943 num Seminário de Verão do Social Sciences Research Council (SSRC 1954). Temos aqui, como 
alternativas já delineadas, trajetos como Processos. Por exemplo, a evolução ou progressão pode ser representada de maneira gráfica, de modo a aumentar a sua compreensibilidade, qual seja:

$$
\mathrm{X} \longrightarrow \mathrm{Y}
$$

enquanto podemos representar a substituição como

$$
\mathrm{X} \longrightarrow \mathrm{I} \longrightarrow \mathrm{Y}
$$

O paralelismo em processos pode-se indicar como

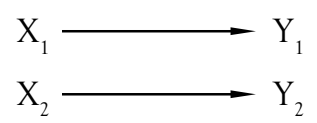

A convergência de fenômenos, por sua vez, pode-se indicar como o seguinte:

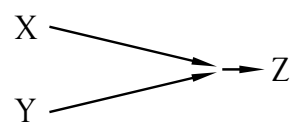

e ainda a divergência:

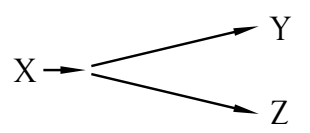

Todas as situações que estamos aqui levando em consideração têm que ser incluídas no elenco de hipóteses alternativas. Para isto, elabora-se uma série de observações em forma de modelos concretos, sobre a relação entre o homem e o ambiente na região, e sistematizamse as pressuposições, as hipóteses e as implicações testáveis contra os dados do mundo real sendo investigado.

A nossa sugestão é de que as hipóteses e implicações sejam colocadas na forma de lógica simbólica, ou lógica formal, não porque as "fórmulas" lógicas ou matemáticas possam ser consideradas mágicas, ou importantes por parecerem abstrusas, mas porque exigem a objetivação de todos os aspectos, às vezes implícitos e escondidos, que precisam ser examinados, sugerindo, ainda, a possibilidade de novas hipóteses alternativas pela manipulação lógica.

O que está envolvida aqui é a manipulação de elementos, tais como os fenômenos e suas inter-relações. É assim que se formulam hipóteses de trabalho, no início da pesquisa: manipulam-se possibilidades a serem investigadas. Também é o caso da formulação de hipóteses de interpretação na fase final do trabalho: manipulam-se os dados levantados em relação a outros fenômenos.

O que é que se pode usar para objetivar tais relações, tornando inteiramente explícitas (portanto apto a ser examinado e reformulado) todas as pressuposições e possibilidades tratadas implícita ou intuitivamente? Já mencionamos: a Lógica Simbólica (Bochenski 1970). Agora vamos ver como funciona.

Pressuposições: conforme já observamos, uma das vantagens de modelos, e os enunciados na forma da Lógica Simbólica são modelos - é que muitas das pressuposições implícitas têm que se tornarem explícitas. Em outros trabalhos citamos a expressão exemplificada por Watson, LeBlanc e Redman (1974) de que

$$
C \supset A \rightarrow B
$$

Ou seja, para estes autores: "dentro de condições C, quando há A então haverá B.” Isto é uma declaração de covariação, mas, registramos, a flecha no enunciado dá a impressão de causalidade. Diríamos que tal expressão significa: "em condições C, A será seguida por B”. Sendo assim, não se trata apenas de covariação, pois implica em causalidade sequencial. Melhor seria reescrever o enunciado da seguinte maneira (estamos substituindo as letras $\mathrm{A}, \mathrm{B}$ e $\mathrm{C}$ porque as usaremos para representar outros conceitos - ver o Glossário de Símbolos anexo a este estudo):

$$
\text { (1a) }
$$

$$
Z \supset X \rightarrow Y \text { ou } Z \supset X \wedge Y
$$

o que nos deixa mais tranquilos, porque podemos ler como "em condições Z, tendo X, também tenderá a haver $Y$ " ou, no segundo caso, "em condições Z, X co-varia com Y".

As nossas pressuposições começam com o axioma de que não existe efeito sem causa (é até uma tautologia), mas com o corolário de que se pode remontar às causas através dos efeitos. Nem sempre é o caso, como a moderna Teoria de Sistemas nos mostra, pois a previsibilidade é apenas probabilística. Entretanto, a "retrovisão" é mais fácil do que a previsão. 
As nossas pressuposições iniciais são quatro: a primeira é que

$$
E \supset B+D+P
$$

Ou seja, a ecologia (E) inclui o ambiente (B), as adaptações (D) e uma população humana (P). As adaptações, por sua vez, são

$$
D \supset R+A+I
$$

ou seja, incluem os recursos (R), as atividades (A) de extração e manutenção, e o instrumental (I).

Seguindo uma formulação de Leslie White, entendemos o instrumental como

$$
I \supset T+C(B)
$$

sendo a combinação da tecnologia $(T)$ com o conhecimento do ambiente $[C(B)]$, ou seja, o ambiente efetivo. Finalmente, consideramos que

$$
\text { Ss }>\operatorname{AI}\left(H_{1}\right)
$$

ou seja, que o sistema sociocultural total Ss é maior do que apenas as atividades e instrumental de um só sítio $\left(H_{1}\right)$. Outras seriam possíveis, e provavelmente serão reconhecidas ao longo dos trabalhos de coleta e análise.

Hipóteses: 1.1 - A distribuição diferencial de recursos e matérias primas através da paisagem resultará numa distribuição diferencial de adaptações ecológicas e de atividades econômicas de extração e manutenção, mesmo de uma mesma população. Portanto, diferenças nas proporções de tipos funcionais de instrumentos não implicam em populações distintas, apenas em atividades distintas:

$$
N \notin(R) \Rightarrow N \notin(\mathrm{Ae})
$$

pelo qual se lê: a distribuição diferencial dos recursos implica na distribuição diferencial das atividades econômicas.

1.2: As casas ou abrigos podem ser diferentes em estrutura, dependendo do tipo de acampamento: semi-permanente ou efêmero. Sendo assim, diferenças no tipo de moradia não significam populações distintas, podendo refletir apenas assentamentos diferentes, relacionados com as atividades.

1.3: Apesar de os aspectos funcionais serem diferentes, a tecnologia de fabricação de instrumentos e da cerâmica e, talvez, até a forma geral de estruturas para abrigo (p. ex., circulares), provavelmente eram iguais, pois estes são aspectos que não dependem, necessariamente, do tipo de atividade, se feita pela mesma população.

1.4.1: Portanto, se as diferenças mostram-se apenas nos aspectos mais ligados à função (forma, tamanho, ângulo, qualidade da matériaprima, etc.) - não na tecnologia empregada (tradição tecnológica de lascamento, pasta, cocção, estilo) -, é realmente provável que se trate de atividades diferentes de uma mesma população.

$$
\text { (7) } \therefore F \pm \epsilon+T \pm \notin \Rightarrow(I+A) \in \Downarrow P \in
$$

O que se lê como: "portanto a função (F) relativamente diferente mais a tradição tecnológica relativamente não diferente implicam em instrumental e atividades diferentes, não implicando, porém, numa população diferente.

1.4.2: Ao contrário, se a tecnologia e os estilos são também diferentes, é relativamente provável que se trate de populações distintas em tempo ou em espaço.

$$
\therefore F \pm \epsilon+T \pm \epsilon \Rightarrow P \in
$$

Ao manipular a nossa lógica através de substituições, alcançamos quatro possibilidades a serem testadas no campo das implicações.

$$
\therefore I \in+T \notin \Rightarrow A \in \Downarrow P \in
$$

se o instrumental for diferente mas não a tradição tecnológica, as atividades são diferentes mas não a população. Entretanto, a segunda nos diz que

$$
\therefore I \in+\mathrm{T} \in \Rightarrow \mathrm{A} \in+\mathrm{P} \in
$$

com o instrumental e a tradição tecnológica diferentes, as atividades e também a população devem ser diferentes.

$$
\therefore I \in+T \in \Rightarrow A \cong+P \cong
$$

Este terceiro caso, em que tanto o instrumental quanto a tradição tecnológica não são diferentes, implica numa mesma população realizando as mesmas atividades.

Finalmente, só para completar o quadro, 


$$
\therefore I \notin+T \in \Rightarrow A \cong+P \in
$$

Com o instrumental igual, mas a tecnologia diferente (embora não nos lembremos de ter visto tal caso, não podemos excluir a sua possibilidade), a implicação é: mesmas atividades, mas desempenhadas por outra população.

É interessante a interação de cientistas com relação a novas técnicas e interpretações. $\mathrm{Na}$ apresentação da versão original deste estudo, em reunião, no Rio de Janeiro, em 1980, a Dra. Dorath Uchoa, da USP, apresentou um exemplo de

$$
I \notin+T \notin+A \sim+P \in
$$

simplesmente não previsto por nós: ela encontrou instrumental não diferente, com tecnologia não diferente em atividades semelhantes praticadas por uma população geneticamente diferente. Um excelente exemplo de aculturação pré-histórica.

2.1: As atividades que produzem instrumentais diferentes devem estar associadas a recursos ou micro-ambientes distintos. Sendo assim, deve-se procurar uma covariação entre fatores (no sentido estatístico usado por Binford e Binford 1966), por um lado e recursos ou microambientes, por outro.

$$
R \in \diamond B \in \Rightarrow A I \in
$$

As implicações desta hipótese e aí

$$
\text { (11a) } \quad \therefore A \cong+I \cong \Rightarrow R \cong B \cong\langle P \notin
$$

pelo qual se lê: "Portanto, atividades e instrumental iguais e semelhantes implicam em recursos e ambientes iguais e semelhantes onde a população não seja diferente", e aí devemos incorporar uma mudança da estratégia de pesquisa, pois somos forçados, para entender o sistema sociocultural pré-histórico da região da nossa pesquisa de salvamento, a procurar micro-ambientes não incluídos na própria área de salvamento (implícito também na forma original da primeira hipótese Equação no. 6).

2.2: Mudanças ambientais devem co-variar com mudanças qualitativas na tecnologia e/ou quantitativas na composição dos fatores. Sendo assim, precisa-se de um subprojeto em que sejam estudadas, especificamente, as mudanças ambientais (climáticas e morfogenéticas) que a região tenha sofrido.

$$
\mathrm{M}(\mathrm{A}) \rightarrow \mathrm{M}_{\mathrm{T}}+\mathrm{M}_{\mathrm{I}} \Rightarrow \mathrm{M}_{\mathrm{K}}
$$

Ou seja, mudanças de atividades equivalem a mudanças de tecnologia e instrumental e implicam em mudanças climáticas e/ou adaptativas e evolutivas.

2.3: Tal estudo ou subprojeto aqui recomendado, embora intimamente ligado ao problema da adaptação e evolução de sistemas socioculturais humanos na região, também se justifica pelo poder preditivo que potencialmente possa proporcionar a órgãos e instituições que operam na região, diminuindo, mesmo que parcialmente, a margem de erro no planejamento destes oriundo de mudanças climáticas cujos ciclos são imperfeitamente conhecidos; e mais: esse estudo ou subprojeto se justifica ainda por levar em consideração um prazo muito maior de conhecimento dos efeitos climáticos, hidrográficos e pedogenéticos de diversas atividades antrópicas na área. Tal conhecimento vai exigir outro projeto interdisciplinar e interinstitucional, de meio alcance em tempo, e de envergadura relativamente grande, mas com a vantagem de poder se articular com pesquisas e análises arqueológicas em procedimento, e com as suas amostras tiradas no campo, juntamente com aquelas.

O que fica implícito aqui é que precisamos estudar a partir de onde o ambiente não faz parte da área de salvamento e a sequência de paleoclimas, para poder interpretar o significado de

$$
\text { (13) }\left(\mathrm{A}+\mathrm{H}_{\mathrm{I}}\right)+\left(\mathrm{I}+\mathrm{H}_{\mathrm{I}}\right) \neq\left(\mathrm{A}+\mathrm{H}_{2}\right)+\left(\mathrm{I}+\mathrm{H}_{2}\right)
$$

ou seja, as atividades e instrumental de um lugar podem ser diferentes dos de outro. Uma proposição como

$$
M_{K} \wedge M_{T}
$$

(Mudanças adaptativas ou evolutivas covariam com mudanças tecnológicas) pode ser demonstrada pelo registro arqueológico, mas precisa de evidências independentes para aumentar a confiança nesta afirmação causal. Um exemplo possível pode ser a seguinte relação de covariação: 


$$
\mathrm{M}_{\mathrm{K}} \wedge \sum\left(\mathrm{M}_{\mathrm{A}}\right)
$$

(Mudanças adaptativas ou evolutivas covariam com a soma das mudanças de atividades).

3.1: Os sítios abertos não conservam mais do que certa gama de resquícios de atividades humanas, especificamente os resultados de atividades com materiais duros e modificações do espaço físico. Os padrões de relações entre esses objetos no espaço, mais a sua análise técnico-funcional, proporcionam elementos extremamente valiosos, se indiretos, sobre atividades humanas. No entanto, se na região existirem grutas ou abrigos secos, a sua investigação deve fazer parte do programa de pesquisa geral, senão do projeto, mesmo estando elas fora da área de salvamento, pois aí poderão fornecer informações sobre (a) tecnologia em matérias-primas perecíveis, (b) a identificação botânica e zoológica de espécies utilizadas, e para o quê, e a (c) dieta, através de restos de ossos e sementes e de fezes.

Precisa-se, assim, realizar uma investigação de tais aspectos, portanto, com verba ou pesquisa complementar. Isto está de acordo com a nossa quarta suposição (Equação 1d). Adicionalmente, podemos mostrar, para salientar a necessidade de se sair de um único meio-ambiente (tal como a várzea ou planície de inundação):

$$
H_{1} \supseteq H_{2} \square H_{1} \pm \supset H_{2}
$$

ou seja, sítio "1" e sítio "2" não fazem parte do mesmo conjunto (isto é, $\mathrm{A}+\mathrm{I}$ e talvez $\mathrm{B}$ diferentes), embora possa haver uma certa sobreposição (interseção) no sentido de semelhança parcial do conteúdo.

Para um exemplo da utilidade de tais estudos, de acordo com a nossa $4^{\text {a }}$ pressuposição (Equação 1c), coisas que podem não ser encontradas na área de salvamento seriam indício de agricultura, pois no Arcaico II de Goiás e Minas Gerais, P. I. Schmitz e O. Dias têm evidências de agricultura (7.000 a 4.000 BP) conforme reportaram no III Seminário Goiano de Arqueologia (Miller 1978/80). Tais informações podiam existir perfeitamente nas grutas usadas para certas atividades, fazendo parte, assim, do acervo sociocultural total embora sem aparecer nos sítios abertos. A nossa interpretação de extensos sítios na várzea, com grande quantidade de instrumentos de pedra, seria outra se tivéssemos evidências de que o mesmo povo praticava a agricultura. Teríamos que somar as evidências de sítios de diversos locais da mesma região:

(17) $\sum[S] \subset T+F\left(A_{1}+A_{2}+\ldots+A_{n}\right) \subset H_{1}+H_{2}+\ldots+H_{n}$

Estando de posse (a) dessas informações, (b) do instrumental associado (c) da distribuição tanto dos recursos dentro da área quanto (d) da distribuição dos fatores instrumentais, estaremos em ótima posição para interpretar, com maior margem de confiança (credibilidade), o seu significado global, em termos de atividades socioculturais e socio-econômicas humanas, e as modificações destas, através do espaço e do tempo, dentro da região a ser afetada.

Com isso, podemos (e) contribuir para conhecimento científico de processos socioculturais de adaptação e evolução dentro do contexto da espécie de ambiente tipificada e especificada pela área de salvamento. Além do seu valor em termos de teoria científica antropológica e especificação histórica, esta informação também tem valor, para o futuro, no planejamento tecno-econômico e socio-econômico para a mesma região.

Como Binford já observou, "os artefatos, tendo o seu contexto funcional primário em subsistemas operacionais diferentes do sistema cultural total, exibirão diferencialmente as semelhanças e diferenças (...) a explanação (...) tem que ser oferecida em termos (...) das características funcionais e estruturais dos sistemas culturais" (Binford 1962), ou seja, uma vez que, há tempo, que a Arqueologia Brasileira vem procurando semelhanças e identidades taxionômicas, agora deve procurar medir e explanar diferenças adaptativas e de atividades (ver a nossa Equação no. 6).

A questão de previsibilidade, bem o sabemos, está eivada de problemas. Já observamos que a previsibilidade na ciência é apenas probabilística. No entanto, existe mais convergência nos fenômenos do Universo do que um cálculo das suas probabilidades, na base da Lei de Ashby (Berrien 1968; Epstein 1986), nos indicaria. É que existem coerções e limitações 
(que não passam de coerções negativas) que ainda não mapeamos - as condições-limites dentro das quais os fenômenos têm maior probabilidade de se manifestar.

A Lógica Simbólica se apresenta aqui como, não uma solução dos nossos problemas, nem uma maneira de apresentar resultados em publicações finais, mas antes como um instrumento de disciplinar as formulações teóricas e de ordenar a sequência lógica de proposições e hipóteses de trabalho, eliciando assim potencialmente novas hipóteses complementares a se procurar no registro arqueológico. A sua utilização está no processo de formular hipóteses de trabalho e hipóteses de interpretação a serem testadas contra novos dados. Não é, porém, um fim da pesquisa, mas apenas um meio de se manipularem os dados no laboratório.
A Lógica Simbólica não é "matematizar o homem" nem diminuir a sua humanidade: é um instrumento metodológico de construção de modelos passível de localizar erros despercebidos, inclusive omissões, no nosso raciocínio, explicitando o que frequentemente permanece implícito. E ainda, como mostramos, tal instrumento é compatível até com pequenos projetos, projetos de salvamento etc., tornando desnecessária qualquer abordagem de empirismo puro que possa redundar em perda de informações, por não examinarem-se possibilidades que estão na nossa frente.

É verdade que se podem expressar em palavras as relações que expressamos acima por meio de "fórmulas" da Lógica Simbólica. Mas as suas relações e possibilidades de novas formulações ficam mais patentes quando colocadas dessa forma mais compacta.

MILLER, T.O. Logic and working hypotheses. Revista do Museu de Arqueologia e Etnologia, São Paulo, 19: 3-12, 2009.

Abstract: After initial theoretical and methodological considerations, the author explores some possibilities of the use of Symbolic Logic as an instrument for the elaboration and verification of the relations between and among phenomena under consideration, manipulating the elements to clarify such relations and to generate new and multiple alternative hypotheses. His conclusions are placed in the context of Archaeology, but are valid for any social science.

Keywords: Logic in scientific method - Generation of multiple hypotheses - Archaeology - Symbolic Logic.

\section{Referências bibliográficas}

BARBOSA, A.S.

1976 Estudos de ecologia cultural no Programa Arqueológico de Goiás. In: Schmitz, P.I.; Barbosa, A.S.; Wüst, I. (Orgs.) Arqueologia de Goiás em 1976. Goiânia: Universidade Católica de Goiás e Instituto Anchietano de Pesquisas: 1-15.

BERRIEN, F.K.

1968 General and social systems. New Brunswick: Rutgers University Press.
BINFORD, L.

1962 Archaeology as Anthropology. American Antiquity, 28: 217-225.

BINFORD, L.; BINFORD, S.

1966 A preliminary analysis of functional variability of the Mousterian of Levallois facies. In: Clark, J.D.; Howell, F.C. (Eds.) American Anthropologist, New Series, 68 (2) Part 2: Recent Studies in Paleoanthropology: 238-295. 
American Anthropologist, New Series, Vol. 68, No. 2, Part 2: Recent Studies in Paleoanthropology (Apr., 1966), pp. 238 . 295 (article consists of 58 pages)

BOCHENSKI, I. M.

1970 A History of Formal Logic. Trad. Ivo Thomas. New York: Chelsea.

BROCHADO, J.P.

1977 Alimentação na Floresta Tropical. Instituto de Filosofia e Ciências Humanas, UFRS, Caderno no. 2.

EPSTEIN, I.

1986 Cibernética. São Paulo: Ática, série Princípios.

GUMERMAN, G.J.

1973 The reconciliation of theory and method in archeology. In: Redman, Ch. (Ed.) Research and theory in current archaeology. New York, Wiley \& Sons: 287-299.

HOLE, F.; HEIZER, R.F.

1969 An introduction to prehistoric archaeology, 1. ed., New York: Holt, Rinehart e Winston.

LIMA, T.A.

1987 Por uma abordagem hipotético-dedutiva na arqueologia de salvamento: o caso da Baia da Ribeira, Angra dos Reis, Rio de Janeiro. Revista do Centro de Estudos e Pesquisas Arqueológicas, Arqueologia, Curitiba, UFPR, 5: 29-33.

MILLER, T.O.

1978a Homem, ambiente e sistema: para uma arqueologia antropológica e intersubjetiva. In: Prous, A. (Ed.) Arquivos do Museu de História Natural - UFMG, III: 179-200.

1978b Lógica e Método na Ciência. In: Antropologia como Ciência: Leituras Didáticas, 1: 4-20. Natal: Mestrado em Antropologia Social da UFRN.

1979 Arqueologia como Ciência. Trabalho apresentado na II Jornada Brasileira de Arqueologia, Rio de Janeiro.
1990 Epistemologia e ética nas ciências antropológicas, Natal: UFRN.

MILLER, T.O. (Coordenador de seminário)

1978-80 O Arcaico do Interior Brasileiro. In: Schmitz, P.I.; Barbosa, A.S.; Ribeiro, M.B. (Eds.) Anuário de divulgação científica, Goiânia: Universidade Católica de Goiás, Temas de Arqueologia Brasileira 2 (6).

1980 Projeto de salvamento arqueológico do Baixo Açu: Represa 'Engenheiro Armando Gonçalves'. Natal: UFRN, Museu "Câmara Cascudo", setor de arqueologia.

READ, D.W.; LeBLANC, S.A.

1978 Descriptive statements, covering laws and theories in archaeology. Current Anthropology, 19 (2): 307-335.

REDMAN, Ch.L.

1973 Introduction. In: Research and theory in current archaeology. New York: Wiley \& Sons.

SSRC (Social Sciences Research Council), "Summer Seminar on Acculturation"

1954 Acculturation: an exploratory formulation. American Anthropologist, 56: 973-1002.

STEWARD, J.

1955a Theory of Culture Change. Urbana: University of Illinois Press.

1955b Civilizaciones de irrigación, un studio comparative. Washington: Unión Panamericana, Manuales Técnicos, II.

WAUCHOPE, R. (Ed.)

1955 Functional and evolucionist implications of the patterning of communities. In: Seminars in archaeology, Society for American Archaeology (American Antiquity), Memoir no. 11.

WATSON, P.J., S.A. LeBLANC; REDMAN , CH.L.

1974 El método científico en arqueologia. Madrid: Alianza.

WITTFOGEL, K.

1957 Oriental Despotism. New Haven: Yale University Press.

\section{Anexo}

Glossário de símbolos usados neste estudo

\section{Substantivos}

$\begin{array}{cl}\text { Símbolo } & \text { Significado } \\ \text { A } & \text { Atividades } \\ \text { J } & \text { Sociedade }\end{array}$

Símbolo
R
F

F
Significado

Recursos

Função 


$\begin{array}{cl}\text { Símbolo } & \text { Significado } \\ \text { B } & \text { Ambiente } \\ \text { K } & \text { Clima } \\ \text { C } & \text { Conhecimento } \\ \text { M } & \text { Mudança } \\ \text { D } & \text { Adaptação (-ões) } \\ \text { P } & \text { População (humana) } \\ \text { E } & \text { Ecologia }\end{array}$

\section{Verbos}

\begin{tabular}{cl} 
Símbolo & \multicolumn{1}{c}{ Significado } \\
$\supset$ & está contido em, dentro de \\
$\supseteq$ & não está em \\
$\subset$ & inclui \\
$\subseteq$ & não inclui \\
$\stackrel{\text { implica em }}{\rightleftharpoons}$ & não implica em \\
$\Downarrow$ & equivale a \\
$\wedge$ & co-varia positivamente com
\end{tabular}

Conjunções e preposições
Símbolo

$\mathrm{S}$

$\mathrm{H}$

$\mathrm{T}$

I

$\cdots$

K

S

Significado
com, mais
então, portanto
ou

Adjetivos e advérbios
Em geral em letra minúscula ou como subscrito. Por exemplo, $K_{\epsilon}=$ "distribuição diferente" ou "diferencial”; enquanto $\mathrm{C}_{\mathrm{b}}=$ "conhecimento ambiental".

$\begin{array}{clc}\text { Símbolo } & \text { Significado } & \text { Símbolo } \\ \text { b } & \text { ambiental } & > \\ \text { e } & \text { econômico(s) } & \sim \\ \text { k } & \text { climático } & = \\ \text { i } & \text { instrumental } & \cong \\ \text { s } & \text { social, sociocultural } & \leq \\ \text { t } & \text { tecnológico } & \geq \\ < & \text { menor de que } & \pm \\ \text { x } & \text { contra, vezes } & :\end{array}$

Significado

Sistema

Sítio arqueológico

Tecnologias (tradição)

Instrumentos, (-al)

etc.

Distribuição

Soma de

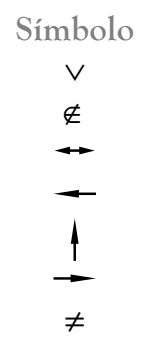

Símbolo

$<$

\rangle $\quad$ Significado
onde
para
embora, apesar de

co-varia negativamente com

é diferente de, não co-varia

é equivalente a

vem de, deriva-se de

sai do contexto; entropia

conduz a, resulta em

não é; é desigual a
Significado

maior de que

semelhante a

igual a

igual e semelhante a

igual ou menor a

igual ou maior a

mais ou menos, relativamente dividido por 